\title{
Alcohol Use and Risky Behaviour: Evidence of Anxiolysis-Disinhibition from a Naturalistic Drinking Study
}

\author{
Charlotte Gayson ${ }^{1}$, Lucy Moss ${ }^{1} \&$ Mark Moss ${ }^{1}$ \\ ${ }^{1}$ Brain, Performance and Nutrition Research Centre, Department of Psychology, Faculty of Health and Life \\ Sciences, Northumbria University, Newcastle upon Tyne, UK \\ Correspondence: Mark Moss, Brain, Performance and Nutrition Research Centre, Department of Psychology, \\ Faculty of Health and Life Sciences, Northumbria University, Newcastle upon Tyne, UK. E-mail: \\ mark.moss@unn.ac.uk
}

Received: November 4, 2014

Accepted: March 23, 2015

Online Published: May 29, 2015

doi:10.5539/ijps.v7n2p129

URL: http://dx.doi.org/10.5539/ijps.v7n2p129

\begin{abstract}
Aims: Alcohol use and intoxication have been widely linked with the incidence of crime and antisocial behaviour. Reduced risk perception following alcohol consumption has been proposed as a possible reason for why people take part in such activities. This study aimed to identify if "intention to act" and "perception of risk" were similarly or differentially affected by alcohol consumed in a natural environment. Furthermore the relationship between amount consumed and degree of impact was investigated.
\end{abstract}

Design: A single factor independent groups design was employed.

Participants: 60 participants aged 18-23 were recruited.

Measures: Participants indicated their likelihood of engagement in a range of acts, and stated how risky they thought each behaviour was via a questionnaire.

Findings: Data analysis revealed a significant effect of alcohol group on reported likelihood of engagement such that likelihood increased with alcohol consumption. However, perceived risk was not subject to any effect, and increased intention to engage in a risky behaviour was not associated with a decreased perception of risk.

Conclusions: These results may provide support for an anxiolysis-disinhibition model of alcohol induced risky behaviour. Certainly the data indicate that cognitive appraisal of the behaviours is not impaired or related to increased engagement.

Keywords: alcohol, intoxication, risk, anxiety

\section{Introduction}

Awareness of the problems associated with alcohol use has increased in recent years. Plant et al. (2006) report that as alcohol consumption rises or falls, the levels of social problems associated with heavy drinking follow the same pattern. Alcohol is related to crime both directly, e.g. drink-driving or drunk and disorderly, and indirectly through offences committed (at least potentially) as a consequence of being intoxicated. The link between alcohol and violent crimes is particularly strong with nearly half of the one million violent crimes annually reported being attributed to alcohol (Office for National Statistics, 2012). Giancola et al. (2009) experimentally assessed the effects of alcohol on aggression in both males and females, finding aggression increased for both sexes after alcohol, but that this increase was more apparent in males. However, research into adolescents does show increasing rates of violence among females too (Odgers et al., 2007), and Graves (2007) concludes that gender differences in serious violent acts are fading. Research employing a large student sample identified a particular problem of increased risk taking when intoxicated, and that these risks covered a variety of domains e.g. sexual, aggression and motoring (O'Brien et al., 2008), and the authors conclude that further research is required to understand this relationship. It is the purpose of the current study to further this understanding.

The psychological factors underpinning the link between alcohol, risk taking and crime have received considerable attention. The presence of a heightened intentional bias after consuming alcohol has been proposed as a possible explanation. This theory states that people believe the behaviour of others is carried out with intent, and that this belief is accentuated by alcohol. As a consequence individuals act in a more aggressive and 
antisocial manner when under the influence of alcohol in response to the perceived intent of others (Bègue et al., 2010). However, explanations grounded in intentional bias describe reactive rather than proactive behaviour and as such are hardly universal - many risky behaviours are self initiated by the drinker. A widely reported alternative explanation of the effects of alcohol on behaviour is Steele and Josephs (1990) Alcohol Myopic Model (AMM) - the proposal that alcohol intoxication restricts the range of cues we are able to process and accurately extract the meaning from. Specifically, alcohol permits one to attend to immediate cues but less able to respond to more peripheral cues, meaning that if the salient cues draw focus to aggression and the peripheral ones inhibit it, alcohol will increase the chance of aggressive behaviour. If this is the case then people may act impulsively; committing risky behaviours in situations for which they do not have an accurate interpretation, however much they may believe that they do.

Distorted perception of the level of risk associated with a given behaviour has also been offered as an explanation of a cause of antisocial or criminal behaviour when intoxicated. Risk perception generally is the subjective assessment of the probability of an accident happening, and evaluations of the negative consequences of an action, (Sjöberg, Moen, \& Rundmo, 2004). It has been hypothesized that those who have been consuming alcohol are susceptible to a reduced sense of risk, increasing the likelihood of them engaging in high risk, often disorderly, behaviour. For example, Banks et al. (2004) found that at legal-to-drive blood alcohol concentrations, alcohol appeared to weaken drivers' ability to detect a crash risk in the environment. However, the incorporation of partial sleep deprivation in the study somewhat confounds the interpretation of a clear alcohol effect. In a different risk-taking arena, gambling research has provided some supporting evidence, with participants who have consumed alcohol being found to persist longer on betting trials and lose more of their original cash than a placebo control group (Kyngdon \& Dickerson, 1999).

Probably the most researched area is the effect of alcohol consumption on sexually risky behaviour. When intoxicated there is an increased likelihood to partake in sex or to conduct indiscriminate forms of risky sex (Thayer et al., 2014). This finding has been found in both between and within subject designs, suggesting that alcohol, rather than other individual differences between those who do and do not drink is the contributory factor (Cooper, 2002). However, the majority of investigators report retrospective rather than prospective data, leaving the evaluation of how participants view something "yet to be done" unreported (e.g. Halpern-Filsher et al., 1996).

Alternative explanations have also been provided regarding the apparent effect of alcohol. Bègue et al. (2009) conducted a naturalistic field experiment and reported that expectancies significantly increased aggressive behaviour, whereas the actual amount of alcohol given did not. Similarly, it has been argued that an individual's belief about the effects of alcohol, both implicit and explicit, may lead to a self-fulfilling prophecy. The behaviour of others reinforces an individuals own beliefs, thus making it seem an acceptable way to act. Subra et al. (2010) showed that the association between alcohol and aggression is so strong that the sheer exposure to alcohol-related primes is enough to automatically activate and increase aggression. This suggests that the association between alcohol and how one should behave is so strong that even words relating to alcohol causes this self fulfilling prophecy to be manifest.

The anxiolysis-disinhibition model (Sayette, 1993) posits that under normal conditions risky behaviour is inhibited by increased subjective anxiety in response to cognitions related to the behaviour. Intoxication with alcohol over-rides or reduces the anxiety response and as such increases risky behaviour via the moderation of a presumably adaptive preventative mechanism. Such a model would suggest that cognitive appraisal of an event need not be impaired or distorted for an aggressive or risky behaviour to be promoted.

The current study aims to improve our understanding of alcohols effects on the likelihood to commit, and the perception of risk associated with risky behaviour through the use of prospective evaluations. The current investigation is also designed to possess high ecological validity through data collection in a bar-room setting where participants have been consuming alcohol (or not) at their leisure. It is hypothesised that alcohol consumption will increase the likelihood of engagement with a range of behaviours classed as risky. The evaluations of perceived level of risk will potentially permit informed suggestions regarding the underlying cause of any such increase.

\section{Method}

\subsection{Design}

A 3 (sober vs moderate vs high alcohol levels) by 2 (gender) independent measures design was employed. The distinction of moderate versus high alcohol groups was made post hoc on a median split of blood alcohol content (BAC) for the alcohol consuming participants. The median value of $0.06 \%$ equated to the UK BAC upper limit 
for driving, and as such made a logical as well as practical point of separation for the groups. The dependent variables derived from the questionnaire were an assessment of how likely students felt they were to engage in the described acts, and the level of risk they associated with the said acts.

\subsection{Participants}

An opportunity sample of thirty male and thirty female students attending a university in the North East of England were recruited in the students' union bar. There was an age range of 18-23 years old $(\mathrm{M}=20.4 \mathrm{SD}=$ 1.6). All participants were volunteers and no compensation was made for their completion of the study. The sample size was based on $\mathrm{G}^{*}$ Power calculations indicating a sample of 64 would produce a power of 0.8 in the presence of an effect size of $\mathrm{F}=.40$. All participants indicated that they were regular drinkers in the bar and that they had been following a usual behaviour pattern in the preceding 24 hours. Potential participants were excluded from the study if they were unable to relay the purpose and process of the study back to the researcher following the provision of the information sheet. This decision was based on the assumption that failure to be able to do so precluded fully informed consent from being possible.

\subsection{Apparatus/Materials}

A 16 item questionnaire consisting of 8 different scenarios (see Table 1 for a complete list) was used. The questionnaire was subdivided into different types of risky behaviours; aggressive (items $2 \& 6$ ), antisocial (items $3 \& 7$ ), moral transgressions (items $4 \& 8$ ), and petty theft (items $1 \& 5$ ).

Table 1. Scenarios used in the questionnaire

Scenario 1 You are walking home and see a pizza delivery moped at the side of the road with some pizzas on the back, but the rider is nowhere to be seen. Would you take the pizzas?

Scenario 2 You are in a bar and someone bumps into you and knocks over your drink. Would you respond aggressively?

Scenario 3 You are out in a public place with friends. Would you take your clothes off and "streak", if dared to?

Scenario 4 You find a purse lying on a table in a club. Would you take the purse and keep any money in it?

Scenario 5 On your way home you see a freestanding road sign that has your name on it. Would you take it?

Scenario 6 Someone pushes into the front of a taxi queue that you have been in for some time. Do you respond aggressively to this?

Scenario 7 You are walking home through a residential area late at night, singing songs with friends. You are asked to keep the noise down by a resident. Would you carry on making noise?

Scenario 8 Someone who you know or believe to be under the age of 18 asks you to buy them cigarettes or alcohol. Would you buy them the product?

For each scenario, the participants indicated how likely they would be to engage in the behaviour $(1=$ Definitely yes to $6=$ Definitely no), and stated how risky they believed the behaviour to be on a scale of 1 (not at all risky) -10 (extremely risky). An Alcoscan AL6000 breathalyzer was also used to measure participants' blood alcohol content according to the manufacturer's instructions.

\subsection{Procedure}

The study gained ethical clearance from the Department of Psychology ethics committee. Participants were recruited in the students union bar, where students had been consuming alcohol (or not) at their leisure. Once informed consent was obtained participants were tested individually to avoid discussion of the questionnaire items. Testing took approximately 15 minutes to complete and participants were asked not to consume alcohol while taking part to ensure the reliability of the breathalyser readings. Upon finishing the questionnaire, participants were asked to provide a sample of breath into the breathalyser. Finally, they were debriefed and thanked for their time.

\section{Results}

\subsection{Questionnaire Evaluation}

To examine the reliability of the scale, coefficient alpha was calculated and produced a value of $\alpha=.716$ indicating the scale to be reliable and further no indication that $\alpha$ would improve if any items were removed. 
To assess the conceptual structure of the eight scenarios loading onto four underlying factors principle components analyses with varimax rotation were performed in SPSS for both intention to engage and ratings of risk. With regard to intention to engage a four factor solution explaining $77 \%$ of the variance was achieved in 5 iterations. The loadings of the items onto the components is presented in Table 2 below and supports the structure of the eight scenarios representing four concepts. As a consequence the ratings for each pair of items corresponding to the four concepts were summed to give total scores that constituted the data for further analysis. With regard to assessment of risk a four factor solution explaining $75 \%$ of the variance was achieved in 7 iterations. The loadings of the items onto the components is presented in table 2 below and further supports the structure of the eight scenarios representing four concepts. As a consequence the ratings for each pair of items corresponding to the four concepts were summed to give total scores that constituted the data for further analysis.

Table 2. Factor loadings for likelihood to engage items and estimated risk on the four conceptualised areas

\begin{tabular}{|c|c|c|c|c|c|c|c|c|}
\hline \multirow{2}{*}{ Scenario } & \multicolumn{4}{|l|}{ Risk } & \multicolumn{4}{|l|}{ Likelihood } \\
\hline & Aggression & Theft & Moral & Antisocial & Aggression & Theft & Moral & Antisocial \\
\hline 1 & & .913 & & & & .813 & & \\
\hline 2 & .869 & & & & .743 & & & \\
\hline 3 & & & & .808 & & & & .563 \\
\hline 4 & & & .870 & & & & .497 & \\
\hline 5 & & .758 & & & & .786 & & \\
\hline 6 & .879 & & & & .829 & & & \\
\hline 7 & & & & .696 & & & & .883 \\
\hline 8 & & & .717 & & & & .945 & \\
\hline
\end{tabular}

\subsection{Alcohol Groups}

The alcohol groups were based on the participants' BAC (\% by volume) readings. All participants tested who produced values of $.01 \%$ or less were classified as constituting the sober group. Participants who possessed BAC values between $.01 \%$ and $.06 \%$ were categorised as the moderate group, and those with BAC readings above $.06 \%$ formed the high alcohol group.

\subsection{Data Analysis}

The data were analysed using the Multivariate Analysis of Variance (MANOVA) procedure in SPSS, with summed evaluations of likelihood to engage forming the dependent variables in one analysis, and the summed evaluations of risk forming the dependent variables in a second Manova. Independent factors for both Manovas were alcohol group and participant gender. Significant Manovas were followed up by Bonferroni corrected univariate analyses of variance (ANOVA) and pairwise comparisons where appropriate, for each behaviour concept in the questionnaire. Partial correlations were also performed to identify if any relationships between $\mathrm{BAC}$ and likelihood to engage in a type of behaviour were potentially mediated by evaluations of risk.

\subsection{Likelihood to Engage in Risky Behaviours}

For the present data the Homogeneity of Covariance Matrices was not significantly violated, Box's $\mathrm{M}=65.655$, $\mathrm{F}(50,5351.928)=1.057, \mathrm{p}=.366$, permitting confident use of MANOVA. The multivariate tests revealed a significant main effect of alcohol group on the likelihood of engaging in the behaviours, Wilks' lambda $=.323, \mathrm{~F}$ $(8,102)=9.688, \mathrm{p}<.001$, and a significant main effect of sex of participant, Wilks' lambda $=.637, \mathrm{~F}(4,51)=$ $7.267, \mathrm{p}<.001$. However, there was no significant group*sex interaction, Wilks's lambda $=.771, \mathrm{~F}(8,102)$ $=.1 .769, \mathrm{p}=.092$. Univariate Anovas were then performed on evaluations for each concept separately and outputs considered in line with the two main effects found to be significant in the Manova. To maintain brevity of reporting only significant effects for these analyses are presented here. Descriptive statistics are presented in Table 3 below. 
Table 3. Mean (and standard deviation) for likeliness of engagement reported by the three alcohol groups on the four behaviour subdivisions

\begin{tabular}{|c|c|c|c|c|}
\hline \multirow{2}{*}{ Behaviour } & \multicolumn{3}{|c|}{ Alcohol Group } & \multirow{2}{*}{ Significant Comparisons } \\
\hline & Sober (1) & Moderate (2) & High (3) & \\
\hline \multirow{2}{*}{ Aggression } & \multirow{2}{*}{$4.07(0.75)$} & \multirow{2}{*}{$3.70(1.10)$} & \multirow{2}{*}{$2.55(0.79)$} & 1 vs $3 * * *$ \\
\hline & & & & 2 vs $3 * * *$ \\
\hline \multirow{3}{*}{ Petty Theft } & \multirow{3}{*}{$5.00(1.11)$} & \multirow{3}{*}{$3.73(1.31)$} & \multirow{3}{*}{$2.23(0.90)$} & 1 vs $2 * *$ \\
\hline & & & & 1 vs $3^{* * *}$ \\
\hline & & & & 2 vs $3 * * *$ \\
\hline \multirow{2}{*}{ Antisocial Behaviour } & \multirow{2}{*}{$4.45(1.03)$} & \multirow{2}{*}{$3.03(1.06)$} & \multirow{2}{*}{$2.35(1.01)$} & 1 vs $2 * * *$ \\
\hline & & & & 1 vs $3 * * *$ \\
\hline \multirow{2}{*}{ Moral Transgression } & \multirow{2}{*}{$5.21(0.86)$} & \multirow{2}{*}{$4.75(0.82)$} & \multirow{2}{*}{$3.63(0.89)$} & 1 vs $3 * * *$ \\
\hline & & & & 2 vs $3 * * *$ \\
\hline
\end{tabular}

Note. All significant comparisons are Bonferroni adjusted; $*<.05, * *<.01, * * * .001$.

\subsection{Aggressive Behaviour}

There was a significant difference between the alcohol groups in terms of their stated likelihood to engage in aggressive behaviour $\mathrm{F}(2,54)=16.305, \mathrm{p}<.001$. Bonferroni corrected post hoc comparisons revealed that the sober group $($ mean $=4.07)$ and moderate group $($ mean $=3.70)$ were significantly less likely to engage in the aggressive behaviour than the high alcohol group (mean $=2.55), \mathrm{p}<.001$ in each case.

\subsection{Petty Theft}

There was a significant difference between the alcohol groups likelihood to engage in Petty theft $\mathrm{F}(2,54)=$ $29.725, \mathrm{p}<.001$. Bonferroni corrected post hoc comparisons revealed that the sober group (mean $=5.00)$ were significantly less likely to engage in the behaviour than both the moderate alcohol group (mean $=3.73) \mathrm{p}=.006$, and the high alcohol group (mean $=2.23), \mathrm{p}<.001$. The moderate group were also significantly less likely to engage in petty theft than the high group, $\mathrm{p}<.001$.

\subsection{Antisocial Behaviour}

There was a significant difference between the alcohol groups likelihood to engage in Antisocial behaviour F (2, $54)=30.603, \mathrm{p}<.001$. Bonferroni corrected post hoc comparisons revealed that the sober group $($ mean $=4.50)$ were significantly less likely than the moderate alcohol group $($ mean $=3.03)$ or the high alcohol group $($ mean $=$ $2.35)$ to engage in antisocial behaviour, $p<.001$ in each case. There was also a significant main effect of sex on likelihood to engage in antisocial behaviour $\mathrm{F}(1,54)=20.030, \mathrm{p}<.001$ with males (mean $=2.70$ significantly more likely to engage than females (mean $=3.86$ ).

\subsection{Moral Transgressions}

There was a significant difference between the alcohol groups likelihood to engage in moral transgressions $\mathrm{F}$ (2, $54)=18.776, \mathrm{p}<.001$. Bonferroni corrected post hoc comparisons revealed that the sober group $($ mean $=5.21)$ and the moderate alcohol group (mean $=4.75)$ were significantly less likely than the high alcohol group (mean $=$ $3.63)$ to engage in this behaviour, $p<.001$ in each case.

\section{Risk Perception}

For risk perception, Box's $\mathrm{M}=58.020, \mathrm{~F}(50,5351.928)=.934, \mathrm{p}=.606$, so the MANOVA can be confidently performed. No significant main effect of alcohol group was found on the composite perceived risk, Wilks's lambda $=.790, \mathrm{~F}(8,102)=1.591, \mathrm{p}=.137$. No significant sex difference was found, Wilks's lambda $=.932, \mathrm{~F}(4$, $51)=.924, \mathrm{p}=.458$. No significant group*sex interaction was found, Wilks's lambda $=.849, \mathrm{~F}(8,102)=1.085$, $\mathrm{p}=.380$. As a consequence, no further analyses were performed. Descriptive statistics are presented in Table 4 below. 
Table 4. Mean (and standard deviation) for risk perception reported by the three alcohol groups on the four behaviour concepts

\begin{tabular}{llll}
\hline Behaviour & $\begin{array}{l}\text { Alcohol Group } \\
\text { Sober }\end{array}$ & Moderate & High \\
\hline Aggression & $5.30(1.66)$ & $5.15(1.55)$ & $4.60(1.17)$ \\
Petty Theft & $5.08(1.62)$ & $4.33(1.66)$ & $3.95(1.16)$ \\
Antisocial Behaviour & $4.35(1.31)$ & $3.95(1.33)$ & $3.47(0.96)$ \\
Moral Transgression & $6.53(1.71)$ & $5.71(1.79)$ & $5.40(1.38)$ \\
\hline
\end{tabular}

\subsection{Partial Correlations}

Correlations between $\mathrm{BAC}$ and likelihood to engage were negative and highly significant for all risky behaviours indicating that as blood alcohol levels increased the likelihood to engage in risky behaviours increased. None of these changed substantially when the relationship with evaluation of risk was partialled out (Table 5).

Table 5. Zero order correlations between BAC and Likelihood and BAC and Risk, plus Partial correlations for relationship between $\mathrm{BAC}$ and likelihood when the relationship with risk is controlled for

\begin{tabular}{llll}
\hline & $\mathrm{r}_{\text {Likelihood.BAC }}$ & $\mathrm{r}_{\text {Risk.BAC }}$ & $\mathrm{r}_{\text {Likelihood.BAC (Risk) }}$ \\
\hline Aggression & $\mathrm{r}(58)=-.627, \mathrm{p}<.001$ & $\mathrm{r}(58)=-.172, \mathrm{p}=.189$ & $\mathrm{r}(57)=-.618, \mathrm{p}<.001$ \\
Antisocial Behaviour & $\mathrm{r}(58)=-.680, \mathrm{p}<.001$ & $\mathrm{r}(58)=-.280, \mathrm{p}=.030$ & $\mathrm{r}(57)=-.646, \mathrm{p}<.001$ \\
Petty Theft & $\mathrm{r}(58)=-.758, \mathrm{p}<.001$ & $\mathrm{r}(58)=-.214, \mathrm{p}=.100$ & $\mathrm{r}(57)=-.762, \mathrm{p}<.001$ \\
Moral Transgressions & $\mathrm{r}(58)=-.618, \mathrm{p}<.001$ & $\mathrm{r}(58)=-.297, \mathrm{p}=.021$ & $\mathrm{r}(57)=-.580, \mathrm{p}<.001$ \\
\hline
\end{tabular}

\section{Discussion}

A significant effect of alcohol consumption on students' likelihood of engagement in all categories of risky behaviour assessed was clearly identified in this study. This prospective data supports previously reported retrospective survey data on the link between students' alcohol use and risk taking (O'Brien et al., 2008). However, this was not matched by a similar effect for the perception of risk indicating that an increase in the likelihood of the behaviour is not a consequence of viewing it as less risky when intoxicated. A significant sex difference was also found for the likelihood of engaging with the behaviours that was equally not matched when considering risk perception. These two factors also clearly acted independently with no interactions evident for any of the dependent variables. Furthermore, the relationship between perceived risk and likelihood to engage did not mediate the relationship between blood alcohol content (BAC) and the likelihood to engage in risky behaviour.

The finding that the likelihood to engage in risky conduct increased with the amount of alcohol consumed is consistent with previous claims that "the amount one drinks should also make a difference ... because alcohol's impairment of perceptual and cognitive functioning increases with dosage" (Steele \& Josephs, 1990, p. 924). With regard to the student population such increases in risky behaviour when intoxicated are well recognised (Wechsler et al., 2000; Hingson et al., 2005; Abbey et al., 2003). Consequently, the current data adds additional support to the relationship between alcohol and disorderly behaviour as it can be concluded that the more alcohol consumed, the more likely students are to engage in risky behaviours - many of which may have negative consequences.

The significant sex difference related to likelihood to engage might also have been anticipated. In the present study males indicated they were more likely than females to engage in the behaviour whether alcohol was involved or not, thus suggesting that males appear to be more risk-oriented than females on the whole. This effect does not appear to be either exaggerated or mitigated by alcohol however as no interactions were evident. 
This runs somewhat counter to previous findings. For example, Giancola et al. (2009) found that aggression increased for both males and females after alcohol consumption, but was more apparent in males. Here however, the impact of gender and alcohol were additive rather than interactive.

No support was found for the possibility that alcohol may reduce risk perception. This somewhat counters Phillips and Ogeil (2010) who concluded that alcohol appeared to influence risk evaluation, and that the encountered problems associated with warning information is consistent with alcohol induced myopia. Participants took longer to make decisions in a situation of high risk when in the high alcohol condition suggesting when intoxicated, more time is needed to process important information. In the current study it would seem that the information was processed and that similar evaluations of risk were arrived at for all behaviours irrespective of alcohol consumption. However, consideration of the mean values indicates that as alcohol consumption increases, ratings of risk reduce and this might suggest a small effect exists but that the sample size in the current study was not sufficient for this to translate to statistical significance. A repetition of this study with a sample size calculated based on the size of effects observed here would allow a further opportunity to investigate the potential presence of this effect. The data from the current study offers partial support to the research reported by Fromme et al. (1997) who demonstrated that alcohol rather than expectancy was the contributing aspect to rating negative outcomes as less likely.

Leigh (1999) proposed a personality approach to the impact of alcohol on behaviour. This was subsequently evidenced by Giancola et al. (2011) who found that alcohol was significantly more likely to increase aggression in participants with a higher aggressive personality score. This presumably would extrapolate to other aspects of risk-taking, impulsivity, and antisocial personality - possibilities that warrant further investigation. In contrast, the situational approach describes how alcohol and potential risks are found in the same environments causing the opportunity for disorderly behaviour to increase. However, the current study might be argued to provide support for a combination of influences. Personality factors might affect who was available for recruitment in the bar, and further those who took up the opportunity. These factors might affect likelihood to engage in risky behaviour, but the consumption of alcohol in the bar environment significantly increased likelihood estimates compared to the non-drinking participants. Importantly however, cognitive explanations based around failure to process risk related information do not stand up here as risk perception was not reduced by alcohol. A more plausible explanation of the alcohol induced increase in risk taking intentions observed here might be drawn from anxiolysis-disinhibition literature (Sayette, 1993; Ito et al., 1996; Phillips \& Giancola, 2008). This model posits that arousal of anxiety occurs in situations where risky or disorderly behaviour might be instigated, and that this anxiety prevents engagement with the risky act. Alcohol may override this arousal of anxiety and thus permit engagement with behaviours that would under sober circumstances be inhibited. In other words (and as reported here) the perception of risk is appraised as the same in circumstances of sobriety and intoxication alike, but the level of engagement increases in proportion to alcohol consumption.

The implications for research showing that the more alcohol consumed, the more likely one would engage in risky behaviours irrespective of risk appraisal are rather complex. Simple predictive relationships cannot be identified and should not be sought, but with alcohol related harm costing the UK an estimated $£ 20$ billion a year (Blair, 2006) the extent of the problems caused by alcohol should not be underestimated. Steele and Josephs (1990) speak of how individual differences alone cannot be the only cause for deciding to engage in certain disorderly behaviours, including the individual reactions to alcohol and personality. This is because alcohol affects behaviours and emotions of everyone, not just those with a special reactivity, and it may affect the same person differently on different days of drinking.

Although providing interesting data, the methodology employed here, i.e. self-report measures, carries some limitations. The relationship between stated intention and actual behaviour patterns is by no means consistent. Sheeran and Abraham (2003) report that in a review of intention - behaviour studies, intention accounted for less than $30 \%$ of the variance in behaviour patterns. Clearly the relationship between likelihood to engage in risky behaviour and actual activity will not be exact. However, with its high ecological validity and reliability, along with use of a breathalyser as measurement of intoxication, the current study has produced intriguing and valid results from which insightful conclusions can be drawn. Specifically, the data show a clear relationship between alcohol consumption and students' expressed likelihood of engaging in a range of risky behaviours. Furthermore, by engaging students who were drinking in a naturalistic setting these data might be more valuable than those collected in a laboratory based study due to possessing greater ecological validity. It would be of considerable value to repeat such a study to include actual risk taking opportunities following alcohol consumption to further understand the relationships involved. Importantly, it is argued that the data here might be explained via the process of anxiolyis-disinhibition. If more data were to confirm such an explanation it would provide impetus for 
developing risk aversion interventions that might be beneficial to the student population and beyond. Further research is warranted into this complex and socially important area.

\section{References}

Banks, S., Catcheside, P., Lack, L., Grunstein, R. R., \& McEvoy, R. D. (2004). Low levels of alcohol impair driving simulator performance and reduced risk perception of crash risk in partially sleep deprived subjects. Sleep, 27(6), 1063-1067.

Bègue, L., Bushman, B. J., Giancola, P. R., Subra, B., \& Rosset, E. (2010). "There is no such thing as an accident," Especially when people are drunk. Personality and Social Psychology Bulletin, 36(10), 1301. http://dx.doi.org/10.1177/0146167210383044

Bègue, L., Subra, B., Arvers, P., Muller, D., Bricout, V., \& Zorman, M. (2009). A message in a bottle: Extrapharmacological effects of alcohol on aggression. Journal of Experimental Social Psychology, 45, 137-142. http://dx.doi.org/10.1016/j.jesp.2008.07.018

Blair, T. (2006). Speech on Healthy Living. Retrieved March 23, 2011, from http://webarchive.nationalarchives. gov.uk/+/http://www.number10.gov.uk/Page9921

Cooper, L. M. (2002). Alcohol use and risky sexual behaviour among college students and youth: Evaluating the evidence. Journal of Studies on Alcohol, S14, 101-117. http://dx.doi.org/10.15288/jsas.2002.s14.101

Fromme, K., Katz, E., \& D’Amico, E. (1997). Effects of alcohol intoxication on the perceived consequences of risk taking. Experimental and Clinical Psychopharmacology, 5(1), 14-23. http://dx.doi.org/10.1037/ 1064-1297.5.1.14

Giancola, P. R., Levinson, C. A., Corman, M. D., Godlaski, A. J., Moriis, D. H., Phillips, J. P., \& Holt, J. C. D. (2009). Men and women, Alcohol and aggression. Experimental and Clinical Psychopharmacology, 17(3), 154-164. http://dx.doi.org/10.1037/a0016385

Giancola, P. R., Parrott, D. J., Silvia, P. J., DeWall, N., Bègue, L., Subra, B., ... \& Bushman, B. J. (2012). The disguise of sobriety: Unveiled by alcohol in persons with an aggressive personality. The Journal of Personality, 80(1), 163-185. http://dx.doi.org/10.1111/j.1467-6494.2011.00726.x

Graves, K. N. (2007). Not always sugar and spice: Expanding theoretical and functional explanations for why females aggress. Aggression and Violent Behaviour, 12(2), 131-140. http://dx.doi.org/10.1016/ j.avb.2004.08.002

Halpern-Felsher, B. L., Millstein, S. G., \& Ellen, J. M. (1996). Relationship of Alcohol Use and Risky Sexual Behavior: A Review and Analysis of Findings. Journal of Adolescent Health, 19, 331-336. http://dx.doi.org/10.1016/S1054-139X(96)00024-9

Ito, T., Miller, N., \& Pollock, V. (1996) Alcohol and aggression: A meta-analysis of the moderating effects of inhibitory cues, triggering events, and self-focussed attention. Psychological Bulletin, 120(1), 60-82. http://dx.doi.org/10.1037/0033-2909.120.1.60

Kyngdon, A., \& Dickerson, M. (1999). An experimental study of the effects of prior alcohol consumption on a simulated gambling activity. Addiction, 94(5), 697-707. http://dx.doi.org/10.1046/j.1360-0443.1999. 9456977.x

Leigh, B. C. (1999). Peril, chance, adventure: Concepts of risk, alcohol use and risky behaviours in young adults. Addiction, 94(3), 371-383. http://dx.doi.org/10.1046/j.1360-0443.1999.9433717.x

Odgers, C. L., Moretti, M. M., Burnette, M. L., Chauhan, P., Waite, D., \& Reppucci, N. D. (2007). A latent variable modelling approach to identifying subtypes of serious and violent female juvenile offenders. Aggressive Behaviour, 33(4), 339-352. http://dx.doi.org/10.1002/ab.20190

Office for National Statistics (ONS). (2012). Crime Statistics, Nature of Crime tables, 2011/12. Retrieved from $\mathrm{http}$ //www.ons.gov.uk/ons/rel/crime-stats/crime-statistics/nature-of-crime-tables--2011-12/index.html

Phillips, J. G., \& Ogeil, R. P. (2010). Alcohol influences the use of decisional support. Psychopharmacology (Berl), 208(4), 603-611. http://dx.doi.org/10.1007/s00213-009-1762-6

Phillips, J. P., \& Giancola, P. R. (2008). Experimentally-induced anxiety attenuates alcohol-related aggression in men. Experimental and Clinical Psychopharmacology, 16, 43-56. http://dx.doi.org/10.1037/ 1064-1297.16.1.43

Plant, M. et al. (2006). Binge Britain-Alcohol and the national response. Oxford University Press: Oxford. 
http://dx.doi.org/10.1093/med/9780199299409.001.0001

Sayette, M. A. (1993). An appraisal disruption model of alcohol's effects of stress responses in social drinkers. Psychological Bulletin, 114, 459-476. http://dx.doi.org/10.1037/0033-2909.114.3.459

Sheeran, P., \& Abraham, C. (2003). Mediator of Moderators: Temporal Stability of Intention and the Intention-Behavior Relation. Personality and Social Psychology Bulletin, 29(2), 205-215. http://dx.doi.org/ $10.1177 / 0146167202239046$

Sjöberg, L., Moen, B., \& Rundmo, T. (2004). Explaining risk perception-An evaluation of the psychometric paradigm in risk perception research. Rotunde: Norway.

Steele, C. M., \& Josephs, R. A. (1990). Alcohol Myopia: Its prized and dangerous effects. American Psychologist, 45(8), 921-933. http://dx.doi.org/10.1037/0003-066X.45.8.921

Subra, B., Muller, D., Bègue, L., Bushman, B. J., \& Delmas, F. (2010). Automatic effects of alcohol and aggressive cues on aggressive thoughts and behaviours. Personality and Social Psychology Bulletin, 36(8), 1052-1057. http://dx.doi.org/10.1177/0146167210374725

Thayer, R. E., Montanaro, E., Weiland, B. J., Callahan, T. J., \& Bryan, A. D. (2014). Exploring the Relationship of Functional Network Connectivity to Latent Trajectories of Alcohol Use and Risky Sex. Current HIV Research, 12(4), 293-300. http://dx.doi.org/10.2174/1570162X12666140721124441

\section{Copyrights}

Copyright for this article is retained by the author(s), with first publication rights granted to the journal.

This is an open-access article distributed under the terms and conditions of the Creative Commons Attribution license (http://creativecommons.org/licenses/by/3.0/). 